\title{
Integrating pedestrian simulation, tracking and event detection for crowd analysis
}

\author{
Matthias Butenuth, Florian Burkert \\ Technische Universität München \\ Remote Sensing Technology \\ \{matthias.butenuth; \\ florian.burkert\} abv. tum. de \\ Florian Schmidt, Stefan Hinz \\ Karlsruhe Institute of Technology \\ Photogrammetry and Remote Sensing \\ \{florian.schmidt; stefan.hinz\}@kit.edu \\ Dirk Hartmann \\ Siemens AG \\ Coroporate Technology \\ hartmann.dirkesiemens.com
}

\author{
Angelika Kneidl, André Borrmann \\ Technische Universität München \\ Computational Modeling and Simulation \\ Group \\ \{kneidl; borrmann\} @bv. tum. de
}

\author{
Beril Sirmacek \\ German Aerospace Center \\ Remote Sensing Institute \\ beril.sirmacek@dlr.de
}

\begin{abstract}
In this paper, an overall framework for crowd analysis is presented. Detection and tracking of pedestrians as well as detection of dense crowds is performed on image sequences to improve simulation models of pedestrian flows. Additionally, graph-based event detection is performed by using Hidden Markov Models on pedestrian trajectories utilizing knowledge from simulations. Experimental results show the benefit of our integrated framework using simulation and real-world data for crowd analysis.
\end{abstract}

\section{Introduction}

We present an interdisciplinary framework for the analysis of crowds in real-world scenarios which integrate benefits of simulation techniques, pedestrian detection and tracking, dense crowd detection and event detection.

Crowd analysis and simulation are emerging fields of research which are motivated by security and monitoring issues in crowded areas. Recent surveys show the achievements and unsolved problems in vision-based crowd analysis, dealing with detection, tracking, occlusion handling, crowd modeling and event inference [16, 32]. The review of Dee and Velastin [7] tries to answer the question "How close are we to solving the problem of automated visual surveillance?", concluding that much work remains in the field of behavior analysis in unstructured and changing environments. Depending on the application, the scale of the analyzed object ranges from individuals [6] to crowds themselves [1].

On the other hand, significant research has been conducted to simulate pedestrian dynamics to predict possible conflict points or bottlenecks. There exists a variety of different simulation models: macroscopic models like network-based models [11] or fluid-dynamics models [14] as well as microscopic models like e.g. the Social Force Model [13] or Cellular Automata [3]. A good overview describing the different approaches and their objectives can be found in Schadschneider et al. [27]. However, the validation of pedestrian simulations is still an open research field. To assure the correctness of simulation results they have to be compared with realworld data captured in the field. A number of small-scale investigations have been carried out already, e.g. Seyfried et al. [29] conducted experiments which examine flows through bottlenecks to gain validation data. Another experiment was conducted by Moussaid et al. [20], where participants walk along a corridor from both directions to examine evading behavior of pedestrians. However, up to now data from real-life situations is not considered very often for validation due to lack of data. In contrast, the framework presented in this paper aims at integrating simulation results and tracking results of crowds from an every-day situation to validate the simulation model.

The utilization of image sequences and video data from airborne sensor platforms for surveillance applications 
such as object tracking has been studied for several years, e.g. [10, 24]. However, research on tracking of people in airborne data is limited. In Miller et al. [19] individuals are detected using corner features, but the results are not satisfying. The work of Reilly et al. [25] received promising detection results including people's shadow in the object model. In our approach, we integrate this information directly in an appearance-based model to deal with coarse image information.

The use of extracted trajectories of pedestrians for event detection has been done in several approaches $[4,5,21$, 31]. A basic tool for the analysis of trajectories are Hidden Markov Models (HMM) [23], which serves for further extensions for event detection and trajectory analysis [21]. Systems for trajectory analysis, region modeling and trajectory mining are well investigated and are still an important topic [5, 31]. However, the basis for such systems are big datasets of only recurring trajectories, as for example at shopping malls or parking lots, which have to be available for each scene of interest. But, there is a lack of prior trajectory datasets at specific big events, where automatic event detection has to be performed spontaneously. Event detection of individual behavior [6] or events which are composed of up to only two people [21] sufficiently copes with behavior of individuals and abandons prior trajectory datasets, but cannot deal with large groups of pedestrians. In contrast, we aim at modeling the behavior of larger groups of people using simulations and tracked pedestrians as input information.

In the next section, we introduce our simulation model applied to a daily-life scenario. Afterwards, the detection and tracking of pedestrians and the detection of dense crowds is presented. In Section 4, we give an overview of our event detection approach using the before derived information. The results using all these developed parts for the analysis of crowds are shown in Section 5 to highlight the benefit of this overall framework.

\section{Simulation of pedestrians}

\subsection{Model description}

To simulate pedestrian crowds, a microscopic approach is used, which consists of several layers:

The time and space discretization is modeled by a cellular automaton, which forms the basic layer.

To model pedestrians' locomotion, a combination of potentials is applied. Each pedestrian is influenced by different forces: a driving force to the destination, repellent forces of obstacles situated on the way to a destination as well as repellent forces of other pedestrian in the scene. These forces are superimposed into one potential field. The corresponding value from the potential field is mapped to each cell of the automation, corresponding to the position of the cell. A detailed description of the potentials approach can be found in Hartmann [12].

The third layer describes the navigation layer, which models the spatial orientation of pedestrians. The layer is implemented as a navigation graph, on which different routing strategies can be applied, e.g. pedestrians who are familiar/are not familiar with a location [17]. An overview of the model setup is shown in Fig. 1.

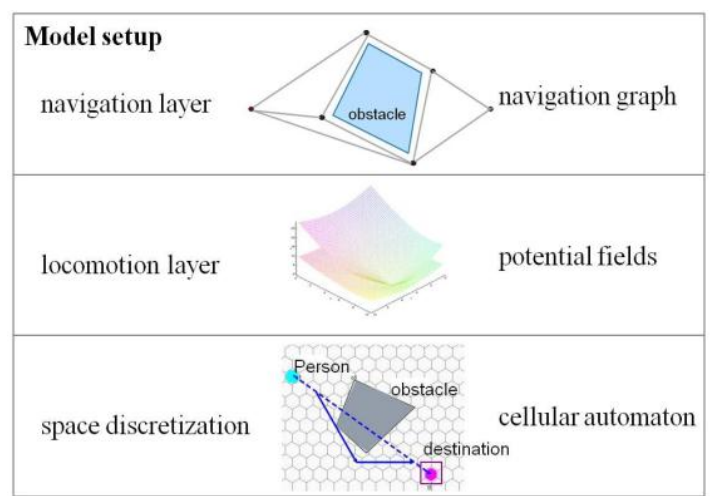

Figure 1: Model setup of the simulation.

\subsection{Scenario}

Each simulated scene is called a scenario. It consists of one or more sources, obstacles and destinations. Pedestrians are generated from sources. The number of generated pedestrians can be adjusted in each time step. Each pedestrian walks towards a destination which has been assigned during generation. Obstacles refer to walls or fences as well as buildings or booths. The scenario of our test case is shown in Fig. 2.

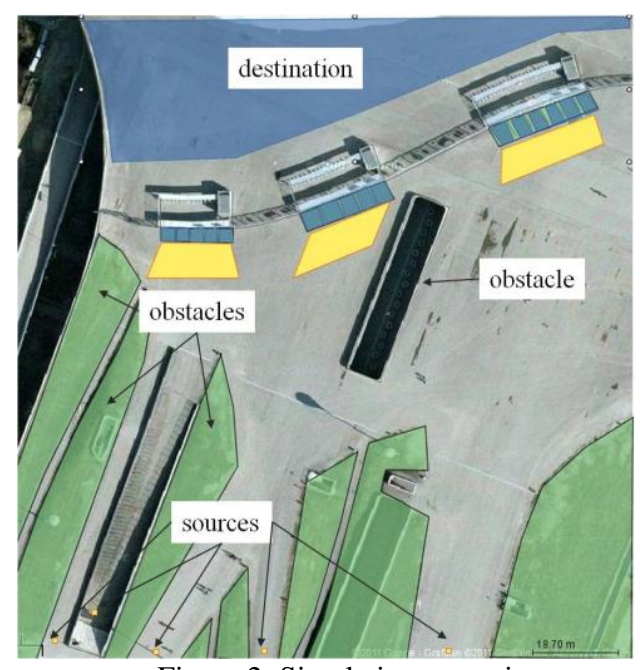

Figure 2: Simulation scenario

\subsection{Simulation setup}

To get realistic start parameters we receive the number 
of pedestrians in the congestion areas through detection of pedestrians as described in the following Section 3.2. The images, to which we compare the simulation results, represent a snapshot of a longer process (cf. Section 5). To get a simulation state comparable to the snapshot, we need an init phase, during which the pedestrians who are generated by the sources in the lower part of the scenario walk towards the crowds in front of the entrances in the upper part (Fig. 2). The following main phase refers to the snapshot from the images, which we compare to the measures from Section 3.

\section{Detection and tracking of pedestrians and crowds}

\subsection{Detection and tracking of pedestrians}

The detection and tracking of pedestrians in aerial image sequences is a challenging task. A single person has a size of just a few pixels and changing atmospheric conditions can lower the visibility (Fig. 3). Furthermore, the number of people can vary from hundreds up to many thousands which all look very much alike. In this section we present the features of our detection and tracking approach which can handle the mentioned challenges. A more detailed description can be found in [28].
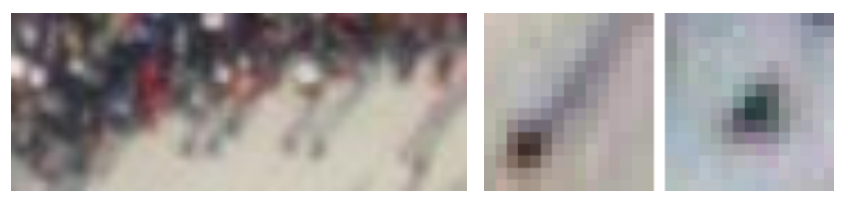

Figure 3: Example of a crowd (left) and a person with and without shadow (center and right) at a pixel size of $0.15 \mathrm{~m}$.

3.1.1. Detection. We utilize an appearance-based approach for object detection since this method has been successfully applied for very small objects, e.g. cars in satellite images [18] or spots in microscopy images [30]. The approach works on single images and can therefore detect small and static objects as opposed to the standard but error-prone methods for moving object detection.

The shadow of a person is a very important cue for detection. We have designed a detector which covers the body of a person and also its potential shadow. A normalization procedure ensures that the shadow will always point in upward direction. We extract color and shape features inside the detection window and pass them to a trained Gentle AdaBoost classifier [9]. It produces a confidence score about the presence of a person at the location of evaluation. By running the customized detector over the region of interest inside an aerial image, we get an independent confidence measure at every pixel position. We then estimate the continuous confidence distribution with a Gaussian kernel. Potential object positions are finally extracted by applying non-maxima suppression and a detection threshold for minimal confidence.

The detection results are the base of the following tracking-by-detection approach. A very low detection threshold ensures that the number of misses stays at a minimum and that the tracking procedure has enough input. The final decision between object and clutter is postponed to the end of the tracking stage, where more information is available.

3.1.2. Tracking. Tracking people in aerial image sequences requires an algorithm that can handle lots of similar objects simultaneously. Further challenges arise due to the low frame rate of e.g. $2 \mathrm{~Hz}$ and deviations in image alignment.

We adapt an iterative Bayesian tracking approach for our application, similar to the one used by [2] to track a large number of flying bats. A single person is described using the following states: position, color and direction of motion. The latter is determined by calculating optical flow between consecutive images. The states of every object are predicted for the next frame with a linear dynamic model. Afterwards the prediction has to be associated with new detection to form tracks. We apply an efficient gating strategy to reduce the number of potential association candidates to a minimum. Each link between prediction and detection is weighted by evaluating their state similarity. The established data association problem is solved in a fast way by using the conservative direct link method of [15]. Objects associated with an observation are updated while unassigned objects are considered lost and are not tracked further. Unassigned observations are marked as new objects. In a final step tracks are rejected if the mean confidence score of all associated detections is below a certain threshold.

3.1.3. Further analysis. The generated trajectories are reliable but rather short. Hence, their potential use for individual motion analysis is still limited. Future improvements aim on generating longer trajectories even in complex situations. However, the results can be used to estimate the total number of persons in the scene and their general motion. The latter can be determined easily given the generated tracklets. The displacement in object position between consecutive frames can be used with the pixel size and the frame rate to calculate the velocity.

The total number of people can be estimated easily from the specific performance range of the detection algorithm:

$$
\begin{gathered}
P=x \cdot P^{\prime}=\frac{T P}{P^{\prime}} / \frac{T P}{P} \cdot P^{\prime}=\frac{\text { correctness }}{\text { completeness }} \cdot P^{\prime} \\
x_{\min } \cdot P^{\prime} \leq P \leq x_{\max } \cdot P^{\prime}
\end{gathered}
$$


The detected number of individuals $P^{\prime}$ can be converted into the true number $P$ by multiplication with the ratio of correctness and completeness. If the variance of the ratio has been determined in advance, it is possible to make a good estimate for the lower and upper bounds of the true number of people in a scene.

\subsection{Detecting dense crowd regions}

The proposed approach presented in the above section will probably fail in dense crowds, because aerial image resolutions do not enable to see each person with sharp contours and details (Fig. 3 left). However, a local change of the color components at the pixels where a person exists can be noticed. Therefore, we develop a dense crowd detection approach depending on local features extracted from chroma bands of the input images. Moreover, dense crowd data can be used to improve the simulation model.

For local feature extraction, we use features from accelerated segment test (FAST) method. The FAST method is especially developed for corner detection purposed by Rosten et al. [26], but the method also detects small regions which are significantly different than their surrounding pixels. We start with converting RGB input image into CIELab color space. CIELab color space bands are preferred since they are able to enhance different colors best and minimize color variances [8]. After transforming, we obtain again three bands as $L$ (intensity value) and $a, b$ (chroma information of the pixels independently from illumination). To detect small regions which have significant color variance compared to their surrounding, we extract FAST features from $a$ and $b$ chroma bands of the image. As local feature, we use $\left(x_{i}, y_{i}\right)$ $i \in\left[1,2, \ldots, K_{i}\right]$ locations which holds FAST features extracted from $a$ and $b$ image bands.

Extracted FAST features will behave as observations of the probability density function (pdf) of the dense crowd locations to be estimated. For dense crowd regions, we assume that more local features should come together. Therefore, knowing the pdf will lead to detection of crowds. For pdf estimation, we benefit from a kernel based density estimation method. Using symmetric Gaussian functions as kernel, the estimated pdf is formed as follows:

$$
p(x, y)=\frac{1}{R} \sum_{i=1}^{K_{i}} \frac{1}{\sqrt{2 \pi \sigma}} \exp \left(-\frac{x-x_{i}^{2}+y-y_{i}^{2}}{2 \sigma}\right)
$$

where $\sigma$ is the bandwidth of Gaussian kernel and $R$ is the normalizing constant to normalize $p(x, y)$ between [0,1]. In kernel based density estimation, the main problem is how to choose the bandwidth of Gaussian kernel for a given test image, since the estimated pdf directly depends on this value. In probability theory, there are several methods to estimate the bandwidth of kernel functions for given observations such as statistical classification or using balloon estimators. Unfortunately, those well-known approaches need high computation time for large input images having very high number of observation points (local features). For this reason, we follow an estimation approach which is slightly different from balloon estimators. First, we pick 20 numbers of random observations (FAST feature locations) to reduce the computation time. For each observation location, we compute the distance to the nearest neighbor observation point. Then, the mean of all distances give us a number $l$. We assume that variance of Gaussian kernel $\left(\sigma^{2}\right)$ should be equal or greater than $l$. In order to guarantee to intersect kernels of two close observations, we assume variance of Gaussian kernels as $5 l$. This automatic kernel bandwidth estimation method makes the algorithm robust to scale and resolution changes. Afterwards, we use Otsu's automatic thresholding method on this pdf to detect regions having high probability values [22]. After thresholding our pdf function in the obtained binary image we eliminate small regions since they cannot indicate large human crowds.

\section{Graph-based event detection using Hidden Markov Models (HMM)}

We perform event detection in image sequences containing large groups of people. Trajectories of tracked pedestrians (cf. Section 3) are used to construct a dynamic pedestrian graph which comprises all detected pedestrians in the scene. Triggered by the existence of edges in the graph, HMM-based analysis of pairwise motion interaction between pedestrians is done [4]. Supported by simulation results (cf. Section 2), the event detection module can be focused on potentially dangerous spots in the scene.

\subsection{Motion model}

Motion interaction between pedestrians is analyzed by inferring the type of motion pattern of two neighboring trajectories, which itself is derived from a set of three motion features.

Three motion features are computed from a pair of neighboring trajectories at each frame, beginning with the second frame of the sequence. The first motion feature is the sum of the velocities of both pedestrians $\sum v_{i j}$. The second motion feature is the variation of the distance between both pedestrians $\Delta d=d_{t} / d_{t-1}$, with $d_{t-1}$ being the distance at frame $t-1$ and $d_{t}$ being the distance at frame $t$. Thus, $\Delta d>1$ at an increasing distance and $\Delta d<1$ at a decreasing distance. The third motion feature is the average pedestrian density in an area with radius $r$ around both pedestrians $\mu\left(n_{i j}\right)$. 
We define six simple pairwise motion patterns which commonly occur at adjacent pedestrians. Pairwise motion patterns are suitable for event detection in crowds, because they focus on motion interaction between pedestrians. In contrast, a single person walking on an open area has no motion interaction to other pedestrians and, thus, is of minor interest for event detection in groups. The six motion patterns are together standing, together queuing, parallel walking, parallel running, diverging, converging, each defined by specific values of the motion features.

\subsection{Dynamic pedestrian graph}

Managing large groups of pedestrians can ideally be performed by constructing a spatio-temporal dynamic graph in which nodes represent pedestrians and edges represent interactions between pedestrians. The dynamic pedestrian graph can change its topology at every frame and is flexible to the number of nodes. The number of edges is kept low by considering only those interactions which take place between directly adjacent pedestrians. This is done by introducing a Gaussian weight function in which the width is depending on the local pedestrian density. The dynamic pedestrian graph is updated at each frame by introducing edges which represent interaction between converging pedestrians or deleting edges which represent interaction between diverging pedestrians.

\subsection{HMM-based event detection}

The temporal behavior of the motion interaction between two pedestrians is evaluated by Hidden Markov Models (HMM) for each edge in the graph throughout the sequence. Usually, HMM are learned offline from realworld training data containing recurring trajectories. However, no training data is available for the monitoring of specific events and the persons cannot be assumed to follow predefined paths. Therefore, we generate synthetic training data which is generated by moving agents. This approach is reliable because the moving agents follow our simple motion model which represents authentic motion interaction of pedestrians. We use about 1000 observations for each of the six motion patterns to train the HMM.

The type of interaction between two pedestrians is inferred by HMM for every edge at every frame using the forward algorithm [23]. We construct a HMM-buffer which internally continues the HMM analysis of one interaction for some frames, even if the corresponding edge is deleted. This may occur when two pedestrians slightly deviate to the left or right and depart from each other awhile. By using the HMM-buffer, the interaction inference will not be interrupted during that time and no fragments of the corresponding interaction arise. The event detection module can deal with a varying number of trajectories of varying length. Trajectories that are too short can be eliminated by applying a threshold for the length. This step is necessary because short trajectory fragments of length 1 or 2 provide no meaningful motion information and increase the computational cost.

\section{Experimental results}

The dataset used for this study is an image sequence taken by an airborne camera platform showing the entrance area of a soccer stadium. The images are taken at a frame rate of $2 \mathrm{~Hz}$, the length of the analyzed image sequence is $8 \mathrm{sec}$, the ground resolution is $0.15 \mathrm{~m}$. For the experimental results, we focus on the area in the south of the stadium gates.

\subsection{Simulation of pedestrians}

The simulation scenario for the stadium dataset is illustrated in Fig. 2. We use measures such as densities and velocities to validate the results of the simulation as well as a visual comparison between the images and the simulation. Until now, the crowds in front of the bottlenecks are only validated by visual checks.

In Fig. 4 simulation snapshots of actual positions and moving directions of simulated pedestrians are shown at the beginning of the tracking phase. In addition, Fig. 5 shows the same snapshots at the end of the tracking phase. These plots can now be compared with the real-world data to check for matches (cf. following sections and Fig. 6).

In our example one can observe the same pattern of pedestrians moving in real vs. pedestrian moving in the simulation. The density within the center dense crowd region can be reproduced by the simulation: The crowd detection data shows a density of 0.81 persons per square meter, whereas the simulation produces a density of 0.79 . Furthermore, the derived velocities from the tracking results of the real-world data are used to improve the model of the simulation velocities.

What can be directly observed from the simulation results is the mismatch of the crowd formation. This can be partly explained by the definition of the repellent obstacle potential. Moreover, until now no queuing effect is implemented within the simulation. To improve the matching between simulation results and real-world data, a further refinement of the simulation model is necessary. But nevertheless, the available real-world data serve a reference data set to improve the simulation model. Furthermore, an adequate comparison measure has to be developed to validate the simulation data with real data.

\subsection{Detection and tracking results of pedestrians and crowds}

The results of the detected dense crowds are visualized in Fig. 6 (red boundaries). The derived results demonstrate 
a reliable detection of dense crowed regions, which are obviously in the front of the gates to the stadium. This information, in particular the dimension and shape of the region, is important to support the simulation model and the detection and tracking of single pedestrians.

The detection and tracking of individuals is focused on the area excluding the detected dense crowds. The quality of the detection and tracking results are evaluated separately with ground truth data. We define a correct detection if its distance to a reference person is below 50 $\mathrm{cm}$ or 3.3 pixel. Our algorithm achieves a correctness of $88 \%$ and a completeness of $36 \%$ (Fig. 6). The main cause for the low completeness is the poor visual quality of the image sequence. The contrast is very low and some thin clouds are passing. Furthermore, lots of people walk in groups which cannot be found by our detector. The correctness is good since there is not much person-like clutter present in the scene. The tracking results are similar. We compare the generated links between consecutive frames with the reference links. Our algorithm achieves a correctness of $89 \%$ and a completeness of $28 \%$. The values reflect the conservative setting of our tracking algorithm and also the previous mentioned difficulties of the images.

We use the detection results to calculate the number of people in the scene as described in section 3.1.3. At first we determine the ratio between correctness and completeness for several different test sequences. It varies between 2.33 and 2.95. We take the median of $P^{\prime}$ for all frames, which is 233 and calculate a lower and upper bound. As a result we estimate the total number of people in the region of interest to lie between 515 and 688. The actual number of people in every frame of the reference data varies between 564 and 597 which approves the proposed estimation method for the evaluated sequence.

\subsection{Event detection results}

The event detection results for pedestrian motion interaction in the test scenario are shown in Fig. 7. The trajectories used for event detection are the tracking results from section 5.2. Fig. 7 shows 252 pedestrian detections (black circles), each of it being part of a trajectory of minimum length 2 and forming a node of the pedestrian interaction graph. In addition, 110 edges are shown which represent motion interactions between pedestrians. Edges are labeled by 6 colors, each of it represents one of the six motion patterns. The detected motion interactions are occurring only between small groups. In most instances the results together walking and converging are delivered. The approach depends on the density of pedestrians. Therefore, more edges in the graph will be constructed when a higher tracking completeness is achieved because then the distances between detected pedestrians are lower.
In that case, a more significant event detection result will be enabled which incorporates larger groups of people.

The simulation results (Fig. 4, Fig. 5) show a location of potential danger in front of the middle entrance. Here, the queue gets close to an obstacle such that passing pedestrians might suffer from a bottleneck situation. Fig. 8 shows a sequence of event detection results based on realworld data in this area. For this result we use manually generated reference trajectories to show the potential of our event detection approach. Arriving pedestrians have to converge and slow down in the narrow area such that congestion occurs, shown by an increased number of yellow edges at a later time. Therefore, the event detection result confirms the simulated dangerous scenario.

\section{Conclusions}

We presented a novel integrated framework for the analysis of crowds including all relevant aspects as simulation, detection and tracking of pedestrians and dense crowds and event detection. The exploitation of the different parts in an overall approach lead to a clear benefit as demonstrated with the real-world scenario.

Our goal for future work is to enhance the system for arbitrary new scenarios, where only a short image sequence is needed for pedestrian tracking and the results are immediately implemented in the update of the simulation model. Moreover, tracked data can help to improve the simulation model. These results allow us to focus on specific locations of potential danger, probably depending on simulated different numbers of pedestrians, and operate only there the visual surveillance and event detection. In addition, the individual parts of the system will be improved, e.g. a better tracking method to track more pedestrians and an enhanced event detection to include more complex events at a higher hierarchical level.

\section{References}

[1] E. Andrade and R. Fisher. Hidden Markov Models for optical flow analysis in crowds. ICPR:460-463, 2006.

[2] M. Betke, D. E. Hirsh, A. Bagchi, N. I. Hristov, N. C. Makris and T. H. Kunz. Tracking large variable numbers of objects in clutter. CVPR:1-8, 2007.

[3] C. Burstedde, K. Klauc., A. Schadschneider and J. Zittartz. Simulation of pedestrian dynamics using a two-dimensional cellular automaton. Physica A: Statistical Mechanics and its Applications, 295(3-4):507-525, 2001.

[4] F. Burkert and M. Butenuth. Event detection based on a pedestrian interaction graph using Hidden Markov Models. Photogrammetric Image Analysis, Springer, LNCS 6952, in print, 2011.

[5] S. Calderara and R. Cucchiara. People Trajectory Mining with Statistical Pattern Recognition. CVPR Workshop, on CD, 2010. 

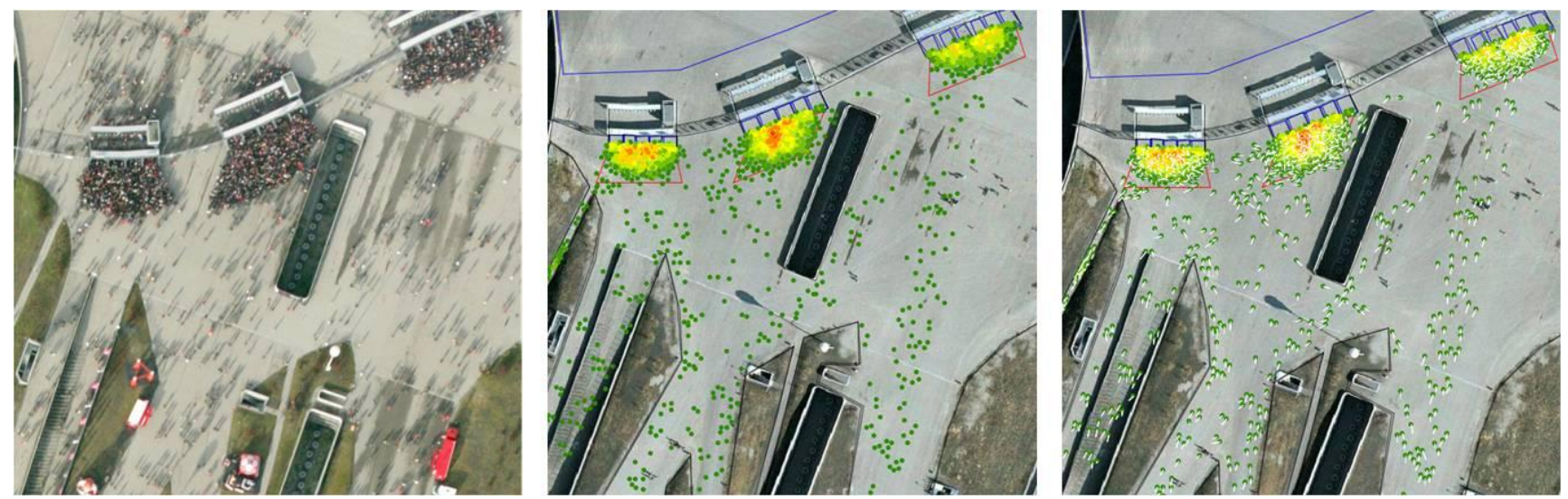

Figure 4: From left to right: Real-world data, actual position simulated pedestrians, moving direction of simulated pedestrians as snapshots at the beginning of the sequence.
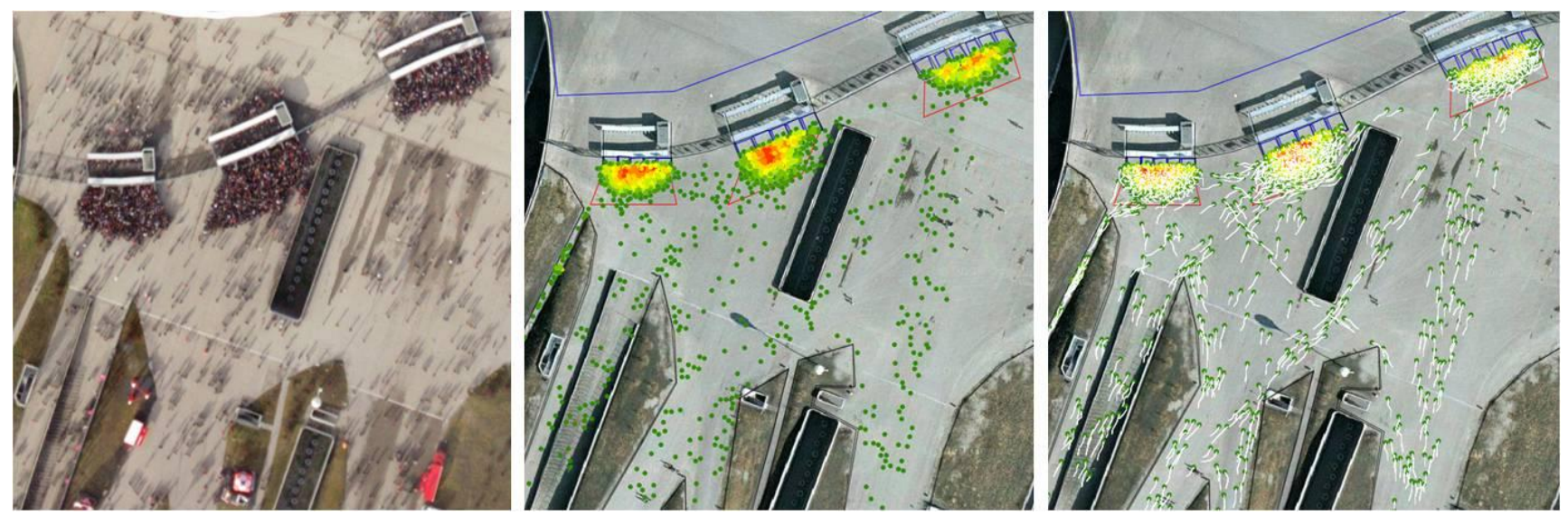

Figure 5: From left to right: Real-world data, actual position simulated pedestrians, moving direction of simulated pedestrians as snapshots at the end of the sequence.

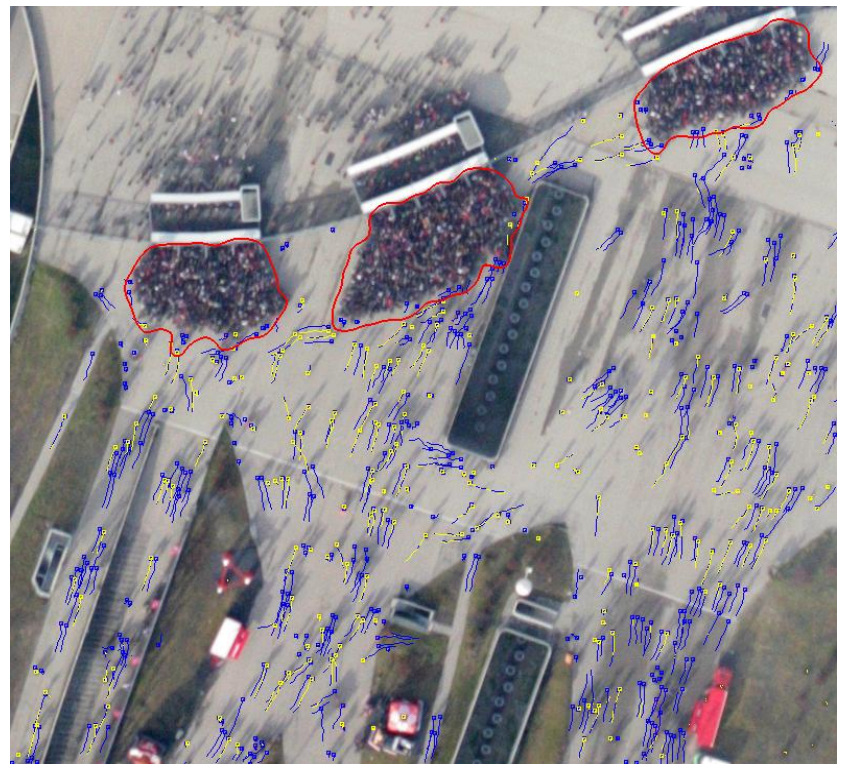

Figure 6: Results of the tracked people (yellow) and the reference data (blue), the tracks are pruned to the last six frames. The detected dense crowds are shown with red boundaries.

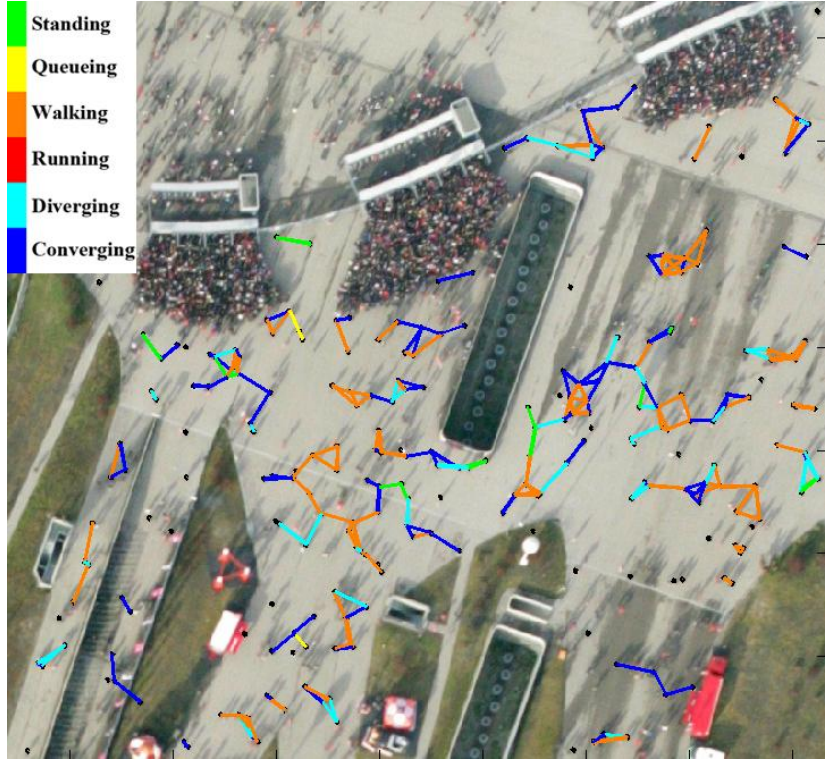

Figure 7: Event detection result based on tracking results from Fig. 6. Colorbar on the left shows the six motion patterns. 

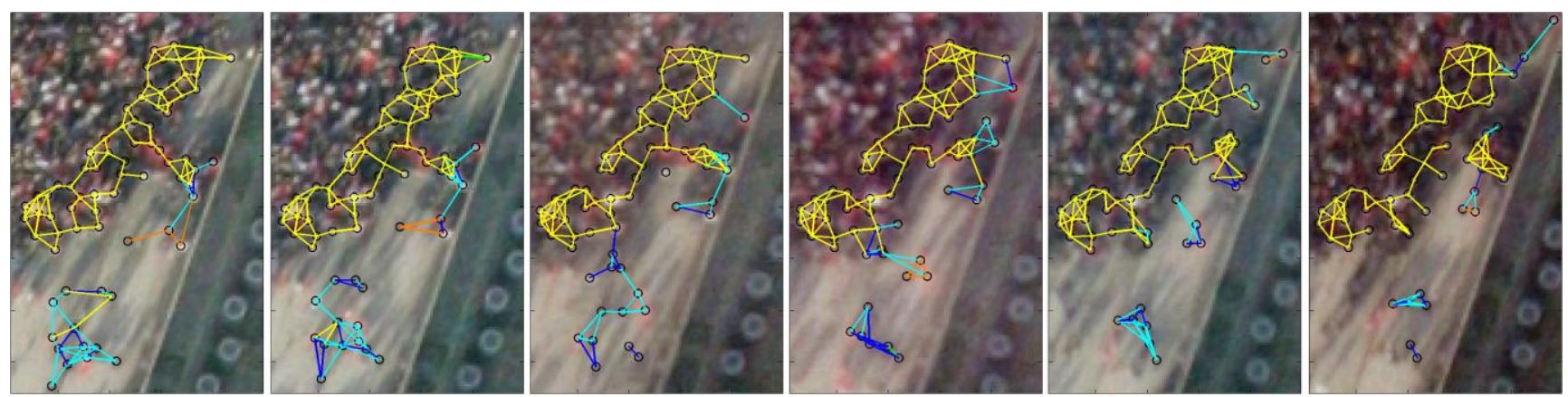

Figure 8: Event detection result showing the border of the dense crowd in front of the middle entrance (frames no. 2, 4, 7, 10, 13 and 16 are shown). The simulation result in Fig. 4 and Fig. 5 shows a potentially dangerous spot at this location.

[6] G. Dalley, X. Wang and W.E.L. Grimson. Event detection using an attention-based tracker. IEEE Workshop PETS, on CD, 2007.

[7] H. M. Dee and S. A. Velastin. How close are we to solving the problem of automated visual surveillance? Machine Vision and Applications, 19(5-6):329-343, 2008.

[8] M. Fairchild. Color appearance models. Addison Wesley, 1998.

[9] J. Friedman, T. Hastie and R. Tibshirani. Additive logistic regression: A statistical view of boosting. The Annals of Statistics, 28(2):337-374, 2000.

[10] H. Grabner, T.T. Nguyen, B. Gruber and H. Bischof. Online boosting-based car detection from aerial images. ISPRS Journal of Photogrammetry and Remote Sensing 63(3):382396, 2008.

[11] H.W. Hamacher, S.A. Tjandra. Mathematical modelling of evacuation problems: A state of the art. Pedestrian and Evacuation Dynamics. Intern. Conference on Pedestrian and Evacuation Dynamics, Springer:227-266, 2002.

[12] D. Hartmann. Adaptive pedestrian dynamics based on geodesics. New Journal of Physics, 12(4): 043032, 2010.

[13] D. Helbing and P. Molnár. Social Force Model for Pedestrian Dynamics. Physical Review E, 51(5):4282-4286, 1995.

[14] L.F. Henderson. On the fluid mechanics of human crowd motion. Transportation Research, 8(6):509-515, 1974.

[15] C. Huang, B. Wu and R. Nevatia. Robust object tracking by hierarchical association of detection responses. ECCV, Springer, LNCS 5303:788-801, 2008.

[16] J.C.S. Jacques, S.R. Musse and C.R. Jung. Crowd Analysis Using Computer Vision Techniques. IEEE Signal Processing Magazine, 9:66-77, 2010.

[17] A. Kneidl, A. Borrmann and D. Hartmann. Generating sparse navigation graphs for microscopic pedestrian simulation models. EG-ICE Workshop, 2011.

[18] J. Leitloff, S. Hinz and U. Stilla. Vehicle detection in very high resolution satellite images of city areas. IEEE Trans. GRS, 48(7):2795-2806, 2010.

[19] A. Miller, P. Babenko, M. Hu and M. Shah. Person tracking in uav video. Multimodal Technologies for Perception of Humans, International Evaluation Workshop. Springer, LNCS 4625:215-220, 2008.

[20] M. Moussaid, D. Helbing, S. Garnier, A. Johansson, M. Combe and G. Theraulaz. Experimental study of the behavioural mechanisms underlying self-organization in human crowds. Royal Society B- Biological Sciences, 276(1668):2755-2762, 2009.

[21] N. M. Oliver, B. Rosario and A. P. Pentland. A Bayesian Computer Vision System for Modeling Human Interactions. IEEE Trans. PAMI, 22(8):831-843, 2000.

[22] N. Otsu. A threshold selection method from gray-level histograms. IEEE Trans. SMC, 9(1):62-66, 1979.

[23] L. R. Rabiner. A Tutorial on Hidden Markov Models and Selected Applications in Speech Recognition. Proceedings of the IEEE, 77(2): 257-286, 1989.

[24] V. Reilly, H. Idrees and M. Shah. Detection and tracking of large number of targets in wide area surveillance. Proceedings of European Conference on Computer Vision. Springer, LNCS 6313:186-99, 2010.

[25] V. Reilly, B. Solmaz, M. Shah. Geometric constraints for human detection in aerial imagery. Proceedings of European Conference on Computer Vision. Springer, LNCS 6316:252-265, 2010.

[26] E. Rosten, R. Porter, T. Drummond. Faster and Better: A machine learning approach to corner detection. IEEE Trans. PAMI, 32(1):105-119, 2010.

[27] A. Schadschneider, W. Klingsch, H. Kluepfel, T. Kretz, C. Rogsch and A. Seyfried. Evacuation dynamics: Empirical results, modeling and applications. Encyclopedia of Complexity and System Science:3142-3176, 2009.

[28] F. Schmidt, S. Hinz. A Scheme for the Detection and Tracking of People Tuned for Aerial Image Sequences. Photogrammetric Image Analysis, Springer, LNCS 6952, in print, 2011.

[29] A. Seyfried, B. Steffen, A. Winkens, T. Rupprecht, M. Boltes and W. Klingsch. Empirical Data for Pedestrian Flow Through Bottlenecks. Traffic and Granular Flow, Springer: 189-199, 2009.

[30] I. Smal, M. Loog, W. Niessen and E. Meijering. Quantitative comparison of spot detection methods in fluorescence microscopy. IEEE Trans. Medical Imaging, 29(2):282-301, 2010.

[31] X. Wang, K. T. Ma, G. Ng and W. E. L. Grimson. Trajectory Analysis and Semantic Region Modeling Using Nonparametric Hierarchical Bayesian Models. International Journal of Computer Vision, in print, DOI 10.1007/s11263011-0459-6, 2011.

[32] B. Zhan, D.N. Monekosso, P. Remagnino, S.A. Velastin and L. Xu. Crowd Analysis: A Survey. Machine Vision and Applications, 19(5-6):345-357, 2008. 